\title{
Informationstransparenz als Chance am Beispiel von Kundenrezensionen in der Automobilindustrie
}

\author{
Ricarda Schlimbach $\mathbb{D} \cdot$ Lukas Schnell $\cdot$ Reza Asghari
}

Eingegangen: 22. Juni 2020 / Angenommen: 30. September 2020 / Online publiziert: 9. Oktober 2020

(C) Der/die Autor(en) 2020

Zusammenfassung Die Informationstransparenz ist zum Spiegelbild der Offenheit von Unternehmen geworden. Ein Beispiel dafür ist die Erfassung und Veröffentlichung von Kundenrezensionen im Internet. Ihre Bedeutung nimmt vor dem Hintergrund der Verlagerung von Kaufentscheidungsprozessen vom stationären Handel ins Internet auch in analog geprägten Branchen wie der Automobilindustrie zu. In der gesamten Prozesskette von der Datenerhebung, über das Text Mining bis hin zur Publikation ergeben sich durch den Aspekt der Offenheit zahlreiche Potentiale, aber auch Risiken. Diese werden zunächst literaturbasiert herausgearbeitet.

Mit Hilfe einer Fallstudie eines international angelegten Projekts für die Feldarbeit und die Verarbeitung von Kundenrezensionen im Automobilsektor, werden Praxiserfahrungen aus Sicht beteiligter Fachexperten zusammengefasst. Im Zuge dessen werden Potentiale, Hürden und Handlungsempfehlungen im Umgang mit Kundenrezensionen hinsichtlich des Aspekts der Offenheit herausgearbeitet. Im Ausblick wird abschließend die wachsende Relevanz der Informationstransparenz diskutiert.

Schlüsselwörter Kundenrezensionen · Informationstransparenz · Offenheit · Kundenintegration

R. Schlimbach $(\bowtie) \cdot R$. Asghari

Entrepreneurship Hub, TU Braunschweig, Rebenring 33, 38106 Braunschweig, Deutschland

E-Mail: ricarda.schlimbach@tu-bs.de

L. Schnell

TU Braunschweig, Braunschweig, Deutschland 


\title{
Information Transparency as an Opportunity on the Example of Customer Reviews in the Automotive Industry
}

\begin{abstract}
The transparency of information has become a reflection of the openness of companies. One example of this is the recording and publication of customer reviews on the Internet. Their importance is increasing against the background of the shift of purchasing decision processes from stationary trade to the Internet, even in analogous sectors such as the automotive industry. In the entire process chain from data collection, text mining all the way to publication, the aspect of openness raises numerous potentials, but also risks. These will first be worked out on the basis of literature.

With the help of a case study of an international project for field work and the processing of customer reviews in the automotive sector, practical experiences are summarized from the perspective of interviewed experts. In this respect chances, hurdles and recommendations for action in handling customer reviews are worked out, while setting a spotlight on the aspect of openness. Finally, the growing relevance of information transparency is discussed in the outlook.
\end{abstract}

Keywords Customer reviews · Transparency of information · Openness · Customer integration

\section{Offenheit im Umgang mit Kundenrezensionen}

Die Digitalisierung bietet neue Möglichkeiten, Innovation mit dem Kunden entlang der Wertschöpfungskette zu gestalten (Abdulrahman und Bach 2013). Im Zuge der Etablierung des Internets als eines der wichtigsten gesellschaftlichen Informationsund Kommunikationsmedien hat sich der Kommunikationsprozess zwischen Unternehmen und Kunden grundlegend verändert: ausgehend von einer eindimensionalen Kommunikation - vom Unternehmen zum Kunden - hin zu einer interaktiven Kommunikation zwischen den Kunden und dem Hersteller (Haug und Küper 2010). Offenheit im Umgang mit Kundenfeedback wird zum Wettbewerbsfaktor (Younis 2015).

Kunden erwarten nicht nur eine hohe Informationstransparenz, sondern wollen auch selbst ihre Erfahrungen, beispielsweise in Form von Kundenrezensionen (KR) teilen und Produkte und Dienstleistungen aus einer aktiven Rolle heraus mitgestalten (Bruhn und Stauss 2009). KR sind im Kontext der Offenheit ein wichtiges Medium, weil sie einerseits aus Konsumentensicht sicherstellen, dass auch kritische Stimmen gehört werden und andererseits Innovationsimpulse für den Hersteller bereithalten (Dettweiler 2018).

In der Literatur gibt es zahlreiche Begriffe für Online-Kundenbewertungen wie „Kundenrezensionen“, „,Kundenartikulation im Internet“, „Online-Kundenfeedback“ oder „Online-Nutzerrezensionen“ (Dettweiler 2018; Charlton 2012), die im Rahmen dieser Arbeit synonym verwendet werden. Die genannten Begriffe bezeichnen eine positive, neutrale oder negative Kommunikation über ein Produkt, eine Dienstleistung, eine Marke oder einen Anbieter, die von Verbrauchern verfasst und online 
veröffentlicht wird (Hennig-Thurau et al. 2004). Online-Kundenbewertungen verkörpern eine besondere Form der elektronischen Mundpropaganda (Wang und Rodgers 2011) und setzen sich standardmäßig aus den folgenden beiden Elementen zusammen: Zum einen wird eine Rezension in Textform verfasst und zum anderen eine numerische Bewertung gegeben (Dettweiler 2018), wobei sich in der Praxis überwiegend eine Skala von ein bis fünf Sternen etabliert hat (Sparks und Browning 2011). Die Freitextnennungen beinhalten Erfahrungen, Einschätzungen und Meinungen zu kundenrelevanten Informationen aus der Kundenperspektive (Hu et al. 2012; Cheung et al. 2012), sodass sie persönliche Kundeneinblicke ermöglichen (Schindler und Bickart 2005).

Von der Datenerhebung, über die Datenverarbeitung, Ideenpriorisierung und $\mathrm{Pu}-$ blikation bis hin zur Umsetzung eines generierten Innovationsportfolios, bildet das Merkmal der Offenheit immer wieder Chancen und Risiken in der Prozesskette. Schon während der Datenerhebung entscheidet die Offenheit des Herstellers über die Quantität und Qualität der einbezogenen Kundenmeinungen. So ermöglicht die Kombination der Datenerfassung aus internen Kundenzufriedenheitsstudien über eigens gesteuerte Plattformen mit externen Quellen (z. B. Social Media) eine größere Datengrundlage und ein breiteres Spektrum an Konsumentenmeinungen gegenüber rein herstellerseitig betriebener Kanäle. Gleichzeitig birgt aber der Einbezug externer Quellen die Gefahr fehlender Kontrollierbarkeit und ermöglicht keine direkte Nachverfolgbarkeit des Kundenanliegens (Seidel 2013; Schieber und Kruse 2014). Auf dem direkten Kanal zum Hersteller zur Abgabe von Feedback können hingegen Anreize für den Kunden geschaffen werden, seine Meinung abzugeben; gleichzeitig verringert die Öffnung der eigenen Kommunikationskanäle das Risiko, dass unzufriedene Kunden ihren Frust anderweitig (online) kundtun. Auch die Offenheit gegenüber verschiedenen Sprachen und Erhebungsmethoden (z. B. strukturierter Online-Fragebogen gegenüber einem Freitextfeld) befördert die Vielfalt des erhobenen Feedbacks, erfordert aber gleichzeitig höhere Anstrengungen in der sich anschließenden spezifischen Datenverarbeitung (Klaas 2019).

Zur systematischen Auswertung der erhobenen KR, wird zumeist Text-MiningSoftware verwendet (Younis 2015). Dabei handelt es sich um eine schon vor der Jahrtausendwende eingesetzte Technik (Usai et al. 2018), die in strukturierter Form mit ausgefeilten statistischen und linguistischen Verfahren relevante Informationen und potentiell nützliches Wissen aus Textdateien musterbasiert analysiert und segmentiert (Akilan 2015; Klaas 2019). Beispielsweise kann die Kundenzufriedenheit initial völlig offen erhoben werden und dann übergeordnet den Kategorien Produkt, Service/Erlebnis oder Prozess und in diesen Kategorien weiteren Unterkategorien oder Handlungsfeldern zugeordnet werden (Seidel 2013). Hierbei wird zwischen dem „Native Coding“, bei dem der Ursprungstext in seiner Originalsprache weiterverarbeitet wird, und dem ,Translated Coding“ unterschieden, bei dem der Ursprungstext zunächst ins Englische übersetzt und dann erst weiter prozessiert wird (Neri und Pettoni 2008; Younis 2015). Die Offenheit in der Nutzung technischer Software für die Übersetzung und das Text Mining reicht hierbei von Open Source Software bis hin zu herstellerseitig eigens entwickelter und streng geschützter Software (Neri und Pettoni 2008). Open Source Lösungen bieten Kostenvorteile, ein hohes Entwicklungstempo und viel Entwickleraustausch, bergen aber gleichzeitig die Gefahr 
des Kontrollverlustes und sind stark von einer aktiven Community abhängig (Heron et al. 2013).

Für eine mögliche Publikation im Internet sollten die erhobenen Kundenmeinungen möglichst originalgetreu belassen werden, um authentisch die tatsächliche Kundenwahrnehmung wider zu spiegeln (Fiedler und Kissling 2019). Aufgrund ihres informativen Charakters aus Nutzerperspektive (Hu et al. 2012), ihrer großen Reichweite (Dettweiler 2018), ihrer Fähigkeit Verbesserungspotenziale im Angebot von Produkten und Dienstleistungen zu erkennen (Iacob et al. 2013), und ihres erkennbar hohen Einflusses auf die Kaufentscheidung der Konsumenten (Li et al. 2013), spielen nutzerseitig generierte Produktbewertungen (Mudambi und Schuff 2010) eine entscheidende Rolle für den Geschäftserfolg (Cheung et al. 2012). Gerade zur Steigerung der Vertrauenswürdigkeit hat die Offenheit eines Unternehmens zur Publikation sowohl positiver als auch negativer Kundenfeedbacks großes Gewicht (Adam 2012 Schieber und Kruse 2014). Aufgrund der sozialen Beziehung in der elektronischen Mundpropaganda schätzen Kunden die Glaubwürdigkeit doppelt so hoch ein wie bei traditioneller Werbung (Aslam et al. 2011; Dettweiler 2018). Das Vertrauen der Verbraucher ist bei Online-Kundenbewertungen sogar bis zu zwölfmal höher gegenüber den vom Hersteller selbst verbreiteten Informationen. Es hat sich gezeigt, dass $85 \%$ der Verbraucher Online-Kundenbewertungen sogar genauso viel Vertrauen entgegenbringen wie persönlichen Empfehlungen (Arevalo 2018). Prognosen antizipieren, dass der Einfluss von Online-Kundenbewertungen weiter zunehmen wird (Pham 2016; Lis und Korchmar 2013). Sie dienen als ein immer relevanter werdendes Marketinginstrument (Abdulrahman und Bach 2013), um Vertrauen bei den Kunden aufzubauen, Aufmerksamkeit zu gewinnen und schließlich zusätzlichen Gewinn zu generieren (Charlton 2012; Arevalo 2018). Marktforschungsinstitute prognostizieren in diesem Kontext steigende Investitionen und eine branchenübergreifend wachsende Relevanz von KR (Ehrhardt 2019; Penaflorida 2019).

Darüber hinaus greifen Unternehmen auf die Ressource des Kunden als Wissensträger und Innovator zurück, um Bedürfnisse mit Innovationspotential aufzuspüren und intern die Ideengenerierung voranzutreiben (Bruhn und Stauss 2009). Im Zuge des „IDEA Mining“ (Schieber und Kruse 2014) fungieren die Kunden als Ideenlieferanten und Gestalter des Innovationsprozesses. Kundenorientierte Innovation ist dabei eine wesentliche Stellschraube für Unternehmen, um auf sich verändernde Rahmenbedingungen und Kundenansprüche zu reagieren. Um Ideen zielgerichtet zu selektieren und intern zu priorisieren, ist die Offenheit im Informationsaustausch erneut ein fundamentaler Baustein. Einerseits sind der offene Austausch von Informationen intern sowie ein funktionierendes Wissensmanagement zwischen voneinander abgegrenzten Abteilungen (z. B. Marktforschung, Strategie und Entwicklung) enorm wichtig, andererseits sollte eine offene Haltung gegenüber neuen Perspektiven der Kunden gegeben sein, selbst wenn diese von der bisher geplanten internen Strategie des Unternehmens abweichen (Schieber und Kruse 2014).

Schon heute greifen eine Vielzahl von Firmen auf das Konzept der Open Innovation für die Gestaltung und Umsetzung des Innovationsportfolios zurück (Adam 2012; Schieber und Kruse 2014). Ergänzend zu den automatisiert und quantitativ generierten Ideen aus KR-Verbesserungsvorschlägen können beispielsweise in 
Hackathons, Co-Creation-Veranstaltungen oder bei (virtuellen) Ideenwettbewerben Ideen mit Innovationspotential diskutiert und qualitativ ausgestaltet werden (Adam 2012). Unternehmen wie DELL oder Starbucks blicken aufgrund dessen auf zahlreiche mittels Kundenideen gelungener Innovationen zurück und verbesserten in der Folge ihre Produkte und das Kundenerlebnis (Schieber und Kruse 2014).

Aus den aufgezeigten Vorteilen durch Offenheit dank Informationstransparenz im Kontext der Kundenmeinungsintegration, resultiert die hohe branchenübergreifende Relevanz von KR, beispielsweise in der Automobilindustrie (Ehrhardt 2019). Diese stellt eine Branche dar, in welcher KR in den letzten Jahren deutlich an Relevanz gewonnen haben. In einer Studie von Ehrhardt (2019) stufen $93 \%$ der Befragten diese als relevant für den Kaufentscheidungsprozess ein. In einer zweiten Studie geben $68 \%$ der Befragten an, ihre Fahrzeugkaufentscheidung auf Rezensionen gestützt zu haben (Data und Marketing Association 2017). Penaflorida (2019) schlussfolgert, dass Informationstransparenz durch im Internet publizierte KR zur Steigerung der Herstellerreputation im Automobilsektor essentiell ist und zu deren Attraktivität für die Kunden beiträgt. Die literaturbasiert herausgearbeiteten Erkenntnisse werden in der folgenden Fallstudie im Kontext der Offenheit durch Informationstransparenz mit Praxiserfahrungen aus einem internationalen Projekt mehrerer beteiligter Unternehmen des Automobilsektors zur Generierung von KR in der Automobilindustrie angereichert.

\section{Fallstudie}

Das hier vorliegende Studiendesign basiert auf einer Fallstudie (Yin 2009) in der Automobilindustrie. Dabei werden Erfahrungen aus einem großen internationalen Verbundprojekt für die Erfassung und Veröffentlichung von KR zwischen drei deutschen Automobilmarken und mehreren beteiligten Dienstleistern und Marktforschungsinstituten herangezogen. Insgesamt umfasst das Projekt die Feldarbeit und das Berichtswesen für über drei Millionen verifizierte Kundenzufriedenheitsbewertungen pro Jahr in rund 20 Sprachen in über 60 Märkten, die weltweit generiert, analysiert und (teilweise) veröffentlicht werden.

Kunden der beteiligten Unternehmen haben die Chance, einen standardisierten Kundenzufriedenheitsfragebogen auszufüllen, wobei sie ihre Gesamtzufriedenheit mit dem Service und mit dem gekauften Produkt auf einer 5-Sterne-Skala und in Form eines Freitextes abgeben können. Die genaue Ausgestaltung des Fragebogens divergiert leicht zwischen den verschiedenen Marken, ist im Kern jedoch inhaltlich und strukturell vergleichbar.

Um die erforderlichen Informationen für die Fallstudie zu generieren, werden verschiedene Datenquellen trianguliert. Ausgangspunkt der Datengenerierung war die im vorangegangenen Abschnitt aufbereitete Ergebnisdarstellung der fundierten Literaturrecherche. Sie bildet einerseits die theoretische Grundlage und dient zum anderen als Referenzwert für die in der Fallstudie erhobenen Ergebnisse. Ergänzend dazu wurden öffentlich zugängliche Informationen (z. B. veröffentlichte KR) analysiert und mit den Erfahrungen der Autoren in verschiedenen KR-Projekten trianguliert. Vier teilstrukturierte Fachexperteninterviews aus drei beteiligten Unternehmen 
im internationalen Verbundprojekt unterstützten die Erhebung von Kontextdaten und komplettieren die umfangreiche Datentriangulation (Yin 2009) mit Erfahrungen aus der Praxis.

Die teilstrukturierten Interviews fokussieren sich auf vier ausgewählte Experten, welche mehrjährige Erfahrungen in den Bereichen KR und Automobilindustrie aufweisen: Die Experten E1 und E2 bekleiden jeweils seit mehreren Jahren leitende Funktionen bei zwei verschiedenen deutschen Automobilmarken (U1 und U2) und verantworten die internationale Durchführung repräsentativer Kundenzufriedenheitsstudien, aus denen KR zur potentiellen Veröffentlichung generiert werden. Experte E3 ist Key Account für den Automobilsektor in einem europaweit agierenden Unternehmen (U3) und bietet Beratung und eine technische Lösung für die Feldarbeit und das Reporting von Kundenbewertungen an. Experte E4 arbeitet bei derselben Automobilmarke (U1) wie Experte E1 und leitet aus verschiedenen Datenquellen Potentiale für Produktinnovationen ab. Tab. 1 gibt einen Überblick hinsichtlich der befragten Experten und ihrer Rolle im Projekt.

Die Verwendung von teilstrukturierten Interviews ermöglichte die flexible Fokussierung von spezifischen Themen und Fragen innerhalb der Interviews (Alam 2002). Damit wurde dem Interviewer die Möglichkeit eingeräumt, Fragen und den Gesprächsverlauf (je Person und Antworten) teilvariabel zu gestalten. Um die Vergleichbarkeit aller Interviews sicherzustellen, wurde ein Gesprächsleitfaden mit 17 themenspezifischen Schlüsselfragen herangezogen und im Gesprächsverlauf auf bis zu 30 Fragen erweitert. Die Vergleichbarkeit aller Interviews bildet die Grundlage für die zusammenfassende Inhaltsanalyse nach Mayring und Fenzl (2019).

Alle Fragen wurden den Experten unabhängig voneinander gestellt und unter der Verwendung von Aufnahmegeräten protokolliert. Die entstehenden Audiodateien wurden anschließend konvertiert und (automatisiert mit manueller Nachbearbeitung) transkribiert. Nach Mayring und Fenzl (2019) erfolgte anschließend die Inhaltszentralisierung in einem dreischrittigen Prozess: erstens der Fokussierung wesentlicher Inhalte, zweitens der Paraphrasierung und drittens der Reduktion. Im Anschluss müssen die Analyseergebnisse mit den gestellten Fragen in Bezug auf Plausibilität und Richtigkeit überprüft werden, wodurch die Objektivität und Reliabilität unterstützt wird. Mit den Ergebnisse können Aussagen kategorienspezifisch erfasst werden (Mayring und Fenzl 2019). Dennoch muss eingeräumt werden, dass die Ergebnisse aus einer kleinen Interviewserie stammen, sodass die Fallstudie nur Anhaltspunkte liefert, aber nicht repräsentativ für die gesamte Automobilindustrie im Umgang mit KR ist.

Die Auswertung zeigt, dass alle befragten Experten große Potentiale in der technischen, fachlichen und prozessualen Öffnung der einzelnen Schritte im Umgang mit KR sehen. Dennoch besteht an verschiedenen Stellen eine große Diskrepanz zwischen der theoretisch möglichen und der tatsächlich realisierten Öffnung im untersuchten Verbundprojekt, weshalb im Folgenden der Istzustand entlang der einzelnen Phasen basierend auf den Expertenaussagen skizziert wird. 


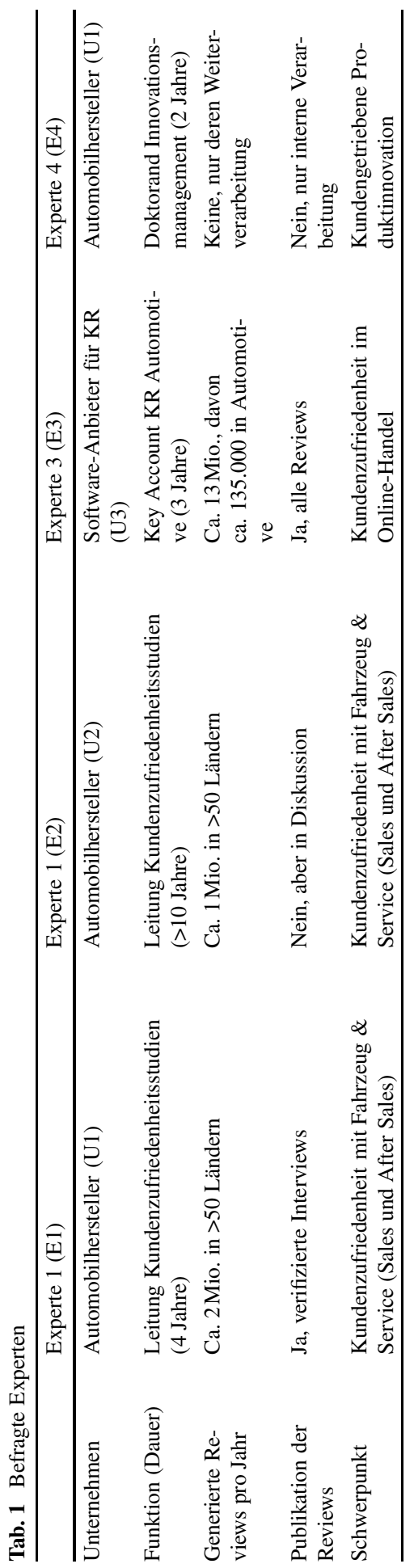




\subsection{Datenerhebung}

Die Unternehmen 1, 2, und 3 ermöglichen jedem verifizierten Kunden die Abgabe eines (anonymisierten oder personalisierten) Reviews durch eine individuelle Einladung kurz nach dem tatsächlichen Kundenkontakt. Sie beabsichtigen damit ,jedem Kunden eine Stimme zu geben" (E2). Dadurch, dass in den Unternehmen 2 und 3 nur verifizierte Kunden zur Abgabe eines Reviews eingeladen werden, verhindern sie sogenannte Fake Reviews (Fiedler und Kissling 2019) vermeintlicher Kunden, welche falsche Rezensionen erstellen, die gar nicht auf tatsächlich gemachten Erfahrungen basieren und daher falsche Informationen streuen. Gleichzeitig entgeht ihnen somit aber auch die Transparenz, Meinungen potentieller (und somit nicht-verifizierter) Kunden zu erhalten, die noch im Kaufentscheidungsprozess stecken oder unzufrieden waren und sich deshalb gegen das Leistungsangebot entscheiden. U1 entschloss sich daher zur weiteren Öffnung des Systems, indem in einigen Märkten bereits nicht-verifizierten Kunden die Möglichkeit gegebenen wird, online proaktiv (ohne nachgewiesenen Handelskontakt) ihre Meinung händlerspezifisch abzugeben. E1 gibt an, dass die KR nicht-verifizierter Kunden erwartungsgemäß im Durchschnitt deutlich schlechter ausfallen als die KR der verifizierten Kunden. Während verifizierte Kunden überwiegend positive KR wie z.B. nach einem Autokauf schreiben, verfassen nicht verifizierte Kunden erfahrungsgemäß häufiger negative KR, da diese in vielen Fällen schon im Kaufentscheidungsprozess (z. B. vom Service) enttäuscht wurden und daher gar nicht erst zu tatsächlichen Kunden werden. Herstellerseitig sei es aber laut E1 extrem wichtig, genau dieses brachliegende Potential schneller aufzuspüren, um dadurch zukünftig weniger potentielle Kunden im Prozess zu verlieren. Aufgrund dieser positiven Erfahrungen ist eine flächendeckende Umsetzung dieser Öffnung in U1 für nicht-verifizierte Kunden in allen Märkten als festgesetzter Standard zukünftig geplant.

Laut den Erfahrungen aller Experten sind die durchschnittlichen Zufriedenheitswerte der repräsentativen verifizierten Kundenmeinungen auf einem sehr hohen Niveau und den durchschnittlichen Bewertungen auf unabhängigen Bewertungsplattformen (z. B. Google, Facebook, Yelp) deutlich überlegen. Als Grund dafür wird der Umstand angeführt, dass von den unternehmensseitig zur Reviewabgabe eingeladenen Kunden die große Mehrheit zufrieden mit dem Service und dem Produkt sei und aufgrund der Einladung zur Reviewabgabe die individuellen Erfahrungen gerne teile. Hingegen werden laut Einschätzung der Experten unabhängige Plattformen häufig erst dann zur Bewertungsabgabe aufgesucht, wenn im Kaufprozess oder während der Nutzung nennenswerte Schwachpunkte aufgetaucht sind, die kommuniziert werden sollen. E1 und E3 erkennen daher das Potential auch von externen Quellen generierte Daten für die weitere Analyse heranzuziehen, um eine noch facettenreichere Datengrundlage zu berücksichtigen. Informationsintransparenz innerhalb der Unternehmen zeigt sich dadurch, dass andere interne Abteilungen, die externe Quellen systematisch verarbeiten, nicht bekannt sind und die technischen Möglichkeiten nie genauer untersucht oder hinterfragt wurden. 


\subsection{Datenverarbeitung}

Zur systematischen Verarbeitung der gesammelten Kundenmeinungen kommt bei den Automobilherstellern U1 und U2 vollautomatisierte Textminingsoftware zum Einsatz, welche die große Masse an Kundenkommentaren nach Lob und Kritik und weiteren vordefinierten Oberkategorien (z. B. Wartezeit im Service, benannte Bauteile im Produkt) segmentiert. Hierdurch ergibt sich das große Potential Stärken und insbesondere aggregierte Schwachstellen herstellerseitig zu überwachen und dann durch eine dedizierte Analyse der entsprechenden Kundenmeinungen konkrete Maßnahmen für angebotene Leistungen oder notwendige Verbesserungen am Produkt abzuleiten. Jedoch erweist sich das Aufsetzen der nötigen Codepläne für die Software als schwierig, denn die Codes werden mehrsprachig benötigt und müssen im richtigen Kontext Aussagen und zahlreiche Synonyme den richtigen Kategorien zuordnen. Dabei sind die Kategorien meist vorbestimmt und statisch, obwohl die Kundenkommentare immer wieder neue Oberkategorien erfordern würden. Eine besondere Herausforderung stellen laut den befragten Experten zusätzlich die vielen verschiedenen Sprachen der Kundentexte dar, weswegen in beiden Unternehmen eine zwischengeschaltete Übersetzungssoftware zur Translation der Offentexte aus der Ursprungssprache ins Englische vor dem tatsächlichen Codieren aber nach dem Bereinigen der personenbezogenen Daten eingesetzt wird. Diese Übersetzungssoftware basiert in U1 und U2 auf einer dienstleisterseitig bereitgestellten Open Source Lösung, weil die entsprechende Software aufgrund dessen eine höhere Übersetzungsgenauigkeit aufweist. Die verwendete Textmining Software wurde hingegen für alle befragten Unternehmen individuell entwickelt und eingekauft und steht nicht öffentlich zur Verfügung. Die Experten wollen dadurch ihre analysierten Daten vor dem Wettbewerb schützen. Gleichzeitig wünschen sich die Befragten mehr Flexibilität und eine höhere Weiterentwicklungsgeschwindigkeit für die benötigte Software.

\subsection{Publikation}

Die Projekterfahrung zeigt, dass eine geplante Informationstransparenz durch ungefilterte Veröffentlichung händler- oder produktspezifischer Kundenkommentare in der Regel zunächst auf großen Widerstand bei Händlern und Entscheidungsträgern stößt. Die Ängste vor negativem und potentiell geschäftsschädigenden Reviews sind groß. Alle beteiligten Unternehmen berichten von einer initialen Abwehrhaltung, weswegen in allen Märkten initial die gesammelten Reviews im internen Berichtsportal zurückgehalten wurden. Erst allmählich zeigte die gründliche Analyse die überwiegend positiven Meinungen in den Kundenkommentaren deren verborgenes Potential und motivierten den Hersteller U1 zur händlerspezifischen Publikation. Auch bei diesem Schritt gab es noch große Befürchtungen seitens der bewerteten Händler, womöglich im Durchschnitt schlechter als die Wettbewerber abzuschneiden. Dies provoziert letztendlich eine noch detaillierte Analyse der eigenen Schwachstellen mit Ableitung von Maßnahmen und wird dadurch seitens der Befragten als Chance eingestuft.

In U1, welches schon seit über zwei Jahren die Informationstransparenz durch veröffentlichte KR forciert, wurde mittlerweile in rund $80 \%$ der studienteilnehmen- 
den Länder die Veröffentlichung der Kundenkommentare (freiwillig) umgesetzt, weil sich die Vorteile gegenüber den Risiken bewährt haben.

Dennoch publizieren derzeit nur zwei der vier befragten Experten die eigens erhobenen Kundenmeinungen im Internet. Bei den anderen beiden Experten handelt es sich daher um eine asymmetrische Informationstransparenz, indem das jeweilige Unternehmen zwar auf eine Vielzahl von Kundenmeinungen Zugriff hat und daraus systematisch Erkenntnisse ableitet, Kunden hingegen keine Möglichkeit bekommen, ihre Erfahrungen öffentlich zu teilen und auf die Einschätzungen anderer Kunden zurückzugreifen. Laut E1 und E3 sei die Veröffentlichung sowohl negativer, als auch positiver KR insofern wichtig, als dass diese vertrauensbildend für die Kunden wirke, ein authentisches Bild des gesamten Spektrums an wahrgenommener Kundenzufriedenheit wiedergebe und das Image der Marke hinsichtlich gesteigerter Transparenz verbessere (E1, E3). Zudem demonstriere die Kommentierung der kritischen Beiträge, dass das Verbesserungspotential ernst genommen wird und tatsächlich zu Verbesserungsmaßnahmen führt, weshalb E3 die zeitnahe Kommentierung (binnen zweier Tage) empfiehlt. Die große Fallzahl überwiegend positiver KR wirke bei den Kunden einerseits vertrauensbildend, wecke aber auch Misstrauen aufgrund der auftretenden Diskrepanz im durchschnittlichen Rating zu den unabhängigen Plattformen. Deshalb werden derzeit in U1 und U2 Möglichkeiten diskutiert, die quantitativ geringer ins Gewicht fallenden Daten von den unabhängigen Plattformen mit in die unternehmensseitig erfassten $\mathrm{KR} \mathrm{zu}$ integrieren und vice versa. Der fachlichen Offenheit bezüglich einer solchen Umsetzung, stehen jedoch noch technische und datenschutzrechtliche Freigabeprozesse entgegen.

\subsection{Ideengenerierung und Verbesserungsmaßnahmen}

Alle Befragten nutzen die generierten Kundenmeinungen zum systematischen Aufspüren interner Verbesserungspotentiale und leiten daraus konkrete Maßnahmen ab. Die Unternehmen 1-3 ordnen dabei jedem Review eindeutig einem Händler zu, bei dem das Produkt gekauft oder der Service erbracht worden ist. Infolgedessen ist es die Aufgabe des spezifischen Händlers, die Kundenanliegen individuell abzuarbeiten und daraus konkrete Maßnahmen abzuleiten. Dieser Prozess ist über alle teilnehmenden Märkte hinweg standardisiert, wird aber in der jeweiligen Landessprache durchgeführt und dokumentiert. Dank eines übergeordneten Berichtssystems, wird der Hersteller jedoch in aggregierter Form über die Maßnahmen der schlechten KR (1-3 Sterne) informiert und kann diese nachverfolgen. Dabei wird auch dokumentiert, wie sich in der Folge die Gesamtzufriedenheit der Kunden eines jeweiligen Händlers im Zeitverlauf verändert. Diese Informationstransparenz sei wichtig, um einerseits herstellerseitig Best Practices ableiten zu können und aus den Verbesserungsvorschlägen zu lernen und zugleich um im Handel den Handlungsdruck zu visualisieren und die Maßnahmenumsetzung zu überwachen (E2). Laut E1 und E4 sei der interne Prozess zur Ideengenerierung und Verarbeitung der Verbesserungsvorschläge hingegen noch zu intransparent. Gerade bei Ideen, die sich auf das Produkt beziehen sei es sehr schwierig die erfassten Meinungen geschickt so zusammen zu fassen, dass sie einer zuständigen Abteilung zur internen Produktoptimierung zugestellt werden könnte. Sprachbarrieren und intransparente Zuständigkeiten ver- 
schärfen diesen Umstand noch. E4 bemängelt, dass bislang offene Schnittstellen fehlen, um auf die gesammelten KR direkt zugreifen zu können. Trotz der Zugehörigkeit zu U1 fehlt hier der offene Austausch zwischen E1 und E4, sodass eine Vielzahl an Kundenanliegen für den internen Innovationsprozess ungenutzt bleibt. Fehlende Informationstransparenz stellt eine große Hürde dar.

\subsection{Umsetzung eines Innovationsportfolios}

E1, E2 und E3 setzen bislang nur inkrementelle Verbesserungen durch die KR auf Einzelhandelsebene um, wohingegen es auf strategischer Ebene noch keine Einspeisung in das herstellerseitige Innovationsportfolio gibt. Dennoch sind alle Unternehmen überzeugt, dass in der Öffnung der Datenschnittstelle zu weiteren internen Abteilungen ein großes Potential liegt. E4 spürt mit seiner Abteilung Innovationspotentiale auf und wertet dazu (meist aggregierte) Marktforschungsergebnisse aus. Das Ziel ist es dabei, schon frühzeitig marktrelevante Ideen zu identifizieren, selektieren und priorisieren und dann in das interne Innovationsportfolio zu integrieren. Deshalb wäre ein direkter Zugriff auf die Daten von U1 und ein Austausch mit E1 für ihn sehr wertvoll.

\subsection{Sollszenario bezüglich der Offenheit von KR in der Prozesskette}

Der zuvor beschriebene Istzustand im Umgang mit KR in der Praxis, zeigt noch viel Potential die Offenheit technisch, fachlich und prozessual auszuweiten. Abb. 1 illustriert daher ein aus den Expertenäußerungen konsolidiertes Sollszenario zur systematischen Verarbeitung der KR. Im Folgenden wird der Prozess unter Nennung

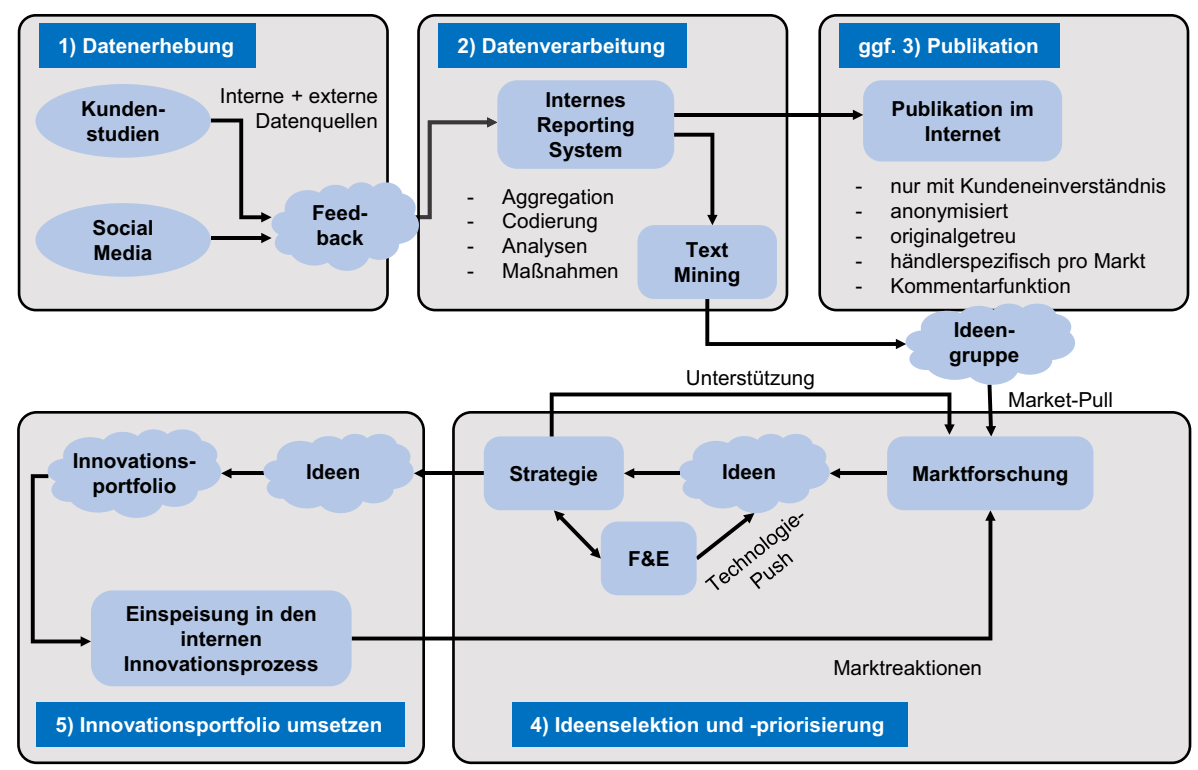

Abb. 1 Skizziertes Sollszenario zur Verarbeitung von Kundenrezensionen 
von konkreten Handlungsempfehlungen beschrieben und es werden Gründe genannt, warum dieser Ablauf derzeit noch nicht vollumfänglich in der Praxis umgesetzt wurde.

Im ersten Schritt der Datenerhebung werden Kundenmeinungen gesammelt. Dazu eignet sich einstimmig laut den Expertenerfahrungen ein möglichst kurzer Fragebogen mit einer 5-Sterne-Skala zur Zufriedenheit mit dem Service und/oder dem Produkt und wenigen, jedoch offen gestellten Fragen, welche dem Kunden die Möglichkeit geben, seine Meinung frei zu formulieren. E4 empfiehlt bei der Verfassung einer Zufriedenheitsbeurteilung eine $\mathrm{zu}$ den Freitextfeldern ergänzende prägnante Überschrift oder vorgelagert kategorisierte Antwortkategorien, um die Reviews im Anschluss besser kategorisieren zu können. Eine Zuordnung der Reviews zu einem bestimmten Kundenkontaktpunkt (z. B. Probefahrtanfrage, Werkstattbesuch, Kaufabschluss, etc.) bereits im Fragebogen ermöglicht einen umfassenden Einblick entlang der gesamten Customer Journey.

Das gesammelte Feedback läuft dann in die interne Berichtsplattform ein. Je nachdem, ob der Kunde der Personalisierung und der Veröffentlichung zugestimmt hat, wird der Kundenkommentar anonymisiert oder personalisiert und verbleibt entweder im unternehmensinternen System zur Weiterverarbeitung oder darf im Internet publiziert werden. Hierbei ist die Offenheit des Kunden zur Veröffentlichung ausschlaggebend. Die Experten betonen, dass es enorm wichtig sei, auf alle negativen (1-3 Sterne) Kommentare möglichst schnell online zu reagieren, selbst wenn der Kommentar nur anonymisiert abgegeben wurde und der Kunde nicht direkt kontaktiert werden kann. Denn durch die unternehmensseitige Reaktion werden die Bemühungen und das Nachverfolgen der Kritik demonstriert, und stärken das Vertrauen in den Service anderer potentieller Kunden. Zur Sicherung größtmöglicher Informationstransparenz werden die Reviews in U1 und U3 ohne Überarbeitung veröffentlicht. Die einzige Ausnahme bilden automatisiert unkenntlich gemachte Angaben zu personenbezogenen Daten, Preisen und Beleidigungen, die aufgrund verschiedener rechtlicher Bestimmungen extrahiert werden müssen.

Im Zuge der Datenverarbeitung und -analyse mit Textmining Software könnte die Offenheit bezüglich Open Source Software und integrierten Wörterbüchern noch weiter gestärkt werden. Durch eine Öffnung der verwendeten technischen Systeme zumindest zwischen den befragten Marken könnte die Weiterentwicklungsgeschwindigkeit und die Qualität der genutzten Software schneller verbessert werden.

Das skizzierte Solszenario zielt darauf ab, die zuvor aus den KR extrahierten Ideen für Produktinnovationen in die Abteilung Strategie weiterzuleiten, um diese in Kooperation mit der Forschung und Entwicklung zu bewerten und ggf. zu verwirklichen. Ausgangspunkt der Ideenbewertung sind vordefinierte Kriterien. Diese zielen auf die Erkennung von Innovationspotentialen ab, welche eine hohe Entwicklungsmöglichkeit zur Realisierung und Einspeisung in den Entwicklungsprozess versprechen. Demzufolge soll das Risiko eines Misserfolges verringert werden. Ideen können dabei überarbeitet, erweitert oder verworfen werden. Aussichtsreiche Innovationsideen werden im Sollszenario im Anschluss an die Beurteilung und ggf. Bearbeitung an das sogenannte Innovationsportfolio übergeben. Das Innovationsportfolio beschreibt ein Set bzw. eine Sammlung von Ideen. Von dort aus können mögliche Produktverbesserungen in den internen Innovationsprozess einge- 
steuert und implementiert werden (Schieber und Kruse 2014). Aktuell fehlt dieser Brückenschlag in allen drei Unternehmen noch, weil die verschiedenen Abteilungen (Marktforschung, Strategie, Entwicklung) nicht wechselseitig auf alle zur Verfügung stehenden Daten zugreifen können.

\subsection{Zukünftige Entwicklungen}

Zukünftige Entwicklungsfelder sehen die Experten in der Erhebung der Kundenrezensionen. Sie wollen diesen Prozess stärker für den Kunden vereinfachen und zu einem Erlebnis werden lassen, indem Sprachassistenzsysteme die Abgabe der eigenen Meinung unkompliziert ermöglichen. Außerdem könnte ein stärker personalisierter Fragebogen und der Einsatz von Chatbots die Interaktion mit dem Kunden verbessern. Neuronale Netze könnten die bislang statisch kodierte Kategorisierung der millionenfachen Offenkommentare der Kunden verbessern, in dem das System selbstlernend weitere Schwerpunktthemen hinzufügt und so neue Handlungsfelder sichtbar macht. Erste Pilotuntersuchungen laufen derzeit mit Partnerunternehmen von U1 und U2. Die Verwendung von Open Source Software wäre ein weiterer möglicher Schritt, um auch das technische System stärker zu öffnen. Allerdings wird dieser Schritt aktuell noch als kritisch angesehen, aufgrund der dazu sehr strengen

Tab. 2 Empfehlungen aus der Praxis

\begin{tabular}{|c|c|c|c|c|}
\hline & Datengenerierung & Datenverarbeitung & Veröffentlichung & Sonstiges \\
\hline \multirow{4}{*}{$\begin{array}{l}\text { Empfeh- } \\
\text { lungen } \\
\text { aus der } \\
\text { Praxis } \\
\text { (Auto- } \\
\text { mobil- } \\
\text { bran- } \\
\text { che) }\end{array}$} & $\begin{array}{l}\text { Allen Kunden } \\
\text { eine Stimme ge- } \\
\text { ben (verfiziert/ } \\
\text { nicht verifiziert) }\end{array}$ & $\begin{array}{l}\text { Intern: Verbesserun- } \\
\text { gen im Service auf } \\
\text { Händlerebene ablei- } \\
\text { ten und umsetzen }\end{array}$ & $\begin{array}{l}\text { Alle Reviews } \\
\text { (positiv/negativ) } \\
\text { veröffentlichen }\end{array}$ & $\begin{array}{l}\text { Mit Pilotprojekt im } \\
\text { geringen Umfang } \\
\text { starten } \rightarrow \text { Ängste } \\
\text { abbauen; Wettbe- } \\
\text { werbsdruck steigern }\end{array}$ \\
\hline & $\begin{array}{l}\text { Wenige, aber } \\
\text { offen gestellte } \\
\text { Fragen } \rightarrow \text { Kun- } \\
\text { de setzt Fokus }\end{array}$ & $\begin{array}{l}\text { Intern: Verbesserun- } \\
\text { gen zum Fahrzeug } \\
\text { auf Herstellerebene } \\
\text { ableiten und umsetzen }\end{array}$ & $\begin{array}{l}\text { Texte (ausgenom- } \\
\text { men rechtliche } \\
\text { Resriktionen) un- } \\
\text { bearbeitet lassen }\end{array}$ & $\begin{array}{l}\text { Kulturspezifika prü- } \\
\text { fen (z. B. Einladungs- } \\
\text { kanal, Mehrsprachig- } \\
\text { keit) }\end{array}$ \\
\hline & $\begin{array}{l}\text { Feedback aktiv } \\
\text { einholen, aber } \\
\text { auch direkte } \\
\text { Kontaktmög- } \\
\text { lichkeiten für } \\
\text { Kunden schaf- } \\
\text { fen }\end{array}$ & $\begin{array}{l}\text { Textminingsoftware } \\
\text { nutzen (native oder } \\
\text { translated coding) }\end{array}$ & $\begin{array}{l}\text { Clusterung nach } \\
\text { Händlern oder } \\
\text { Bauteilen }\end{array}$ & $\begin{array}{l}\text { Best Practice Work- } \\
\text { shops umsetzen }\end{array}$ \\
\hline & $\begin{array}{l}\text { KR entlang der } \\
\text { gesamten Cus- } \\
\text { tomer Journey }\end{array}$ & $\begin{array}{l}\text { Reaktion binnen } \\
\text { zweier Tage zum } \\
\text { Kunden }\end{array}$ & $\begin{array}{l}\text { Schnelle öffent- } \\
\text { liche Kommen- } \\
\text { tierung kritischer } \\
\text { KR }\end{array}$ & $\begin{array}{l}\text { Verbesserte (techn.) } \\
\text { Optionen integrieren } \\
\text { (z. B. Chatbots, AI, } \\
\text { etc.) }\end{array}$ \\
\hline $\begin{array}{l}\text { Benefits } \\
\text { dank } \\
\text { Infor- } \\
\text { mati- } \\
\text { ons- } \\
\text { transpa- } \\
\text { renz }\end{array}$ & $\begin{array}{l}\text { Transparent } \\
\text { sein } \\
\text { Vertrauen schaf- } \\
\text { fen } \\
\text { Direkten Kanal } \\
\text { zum Hersteller } \\
\text { bieten }\end{array}$ & $\begin{array}{l}\text { Kundengetriebene } \\
\text { Verbesserungsprozes- } \\
\text { se und Innovationspo- } \\
\text { tential } \\
\text { Vielfältige Analysen } \\
\text { Intern transparente } \\
\text { Datengrundlage }\end{array}$ & $\begin{array}{l}\text { Integriertes Pro- } \\
\text { duktmarketing } \\
\text { Imageverbesserung } \\
\text { \& Authentizität } \\
\text { Offener Wettbe- } \\
\text { werb } \\
\text { Entscheidungshilfe }\end{array}$ & $\begin{array}{l}\text { Hohe Marktakzep- } \\
\text { tanz im Markt } \\
\text { Zufriedene Kunden } \\
\text { Kontinuierliche } \\
\text { Weiterentwicklung } \\
\text { der identifizierten } \\
\text { Schwachstellen }\end{array}$ \\
\hline
\end{tabular}


und langwierigen (IT- und datenschutzrechtlichen) Freigabeprozesse in den beteiligten Unternehmen.

Tab. 2 fasst Empfehlungen aus der Praxis zusammen, die mit Hilfe der befragten Experten generiert werden konnten. Zudem stellt sie die erreichten Vorteile durch Informationstransparenz im Umgang mit KR dar.

\section{Fazit}

Es ist auch in der Automobilindustrie wettbewerbsentscheidend geworden, Offenheit in der gesamten Prozesskette im Umgang mit KR zu generieren. Die durch Informationstransparenz realisierte Offenheit wirkt sich einerseits vertrauensbildend und kaufentscheidungsunterstützend auf den Kunden aus und ermöglicht andererseits bei systematischer Auswertung das herstellerseitige Potential, Kundenbedürfnisse frühzeitig zu erkennen und in den internen Innovationsprozess zu integrieren. Bei einer hohen Kundenzufriedenheit oder einem kundenfreundlichen Umgang im Falle geäußerter Kritik, fungiert diese Transparenz mit hoher Reichweite zusätzlich als kundengetriebenes Produktmarketing.

Um im Wettbewerb künftig erfolgreich zu bleiben, ist die Offenheit hinsichtlich der Informationstransparenz ergo zum neuen Standard geworden (Younis 2015). Es ist daher zu erwarten, dass sich zukünftig der Unternehmenserfolg noch stärker über die Transparenz von Informationen definiert. Infolgedessen gewinnt die zunehmende öffentliche Erfassung und Bereitstellung kundengenerierter Meinungen für Produkte und Services eines Unternehmens an Relevanz, indem sie die Kunden in den gesamten Wertschöpfungsprozess integrieren. Erfahrene Kunden fungieren als Markenbotschafter für potentielle Kunden und geben darüber hinaus intern und extern wertvolle Impulse für mögliche Prozess- und Produktverbesserungen. Somit resultiert eine Verschiebung der Push- zur Pull-Orientierung eines Unternehmens.

Das Bewusstsein für die Chancen dieser offenen Interaktion mit (potentiellen) Kunden mit hoher beidseitiger Informationstransparenz könnte darüber hinaus zur weiteren Öffnung beteiligter Unternehmen führen, beispielsweise in Form von mehr Open Source Software oder den verstärkten Einsatz von ergänzenden Open Innovation Veranstaltungen. Interne und externe Informationstransparenz ist dafür ein Schlüsselfaktor. Die Expertenmeinungen am Beispiel von KR zeigen deutlich, dass in der Praxis derzeit ein Umdenken zugunsten stärkerer Öffnung stattfindet.

Funding Open Access funding enabled and organized by Projekt DEAL.

Open Access Dieser Artikel wird unter der Creative Commons Namensnennung 4.0 International Lizenz veröffentlicht, welche die Nutzung, Vervielfältigung, Bearbeitung, Verbreitung und Wiedergabe in jeglichem Medium und Format erlaubt, sofern Sie den/die ursprünglichen Autor(en) und die Quelle ordnungsgemäß nennen, einen Link zur Creative Commons Lizenz beifügen und angeben, ob Änderungen vorgenommen wurden.

Die in diesem Artikel enthaltenen Bilder und sonstiges Drittmaterial unterliegen ebenfalls der genannten Creative Commons Lizenz, sofern sich aus der Abbildungslegende nichts anderes ergibt. Sofern das betreffende Material nicht unter der genannten Creative Commons Lizenz steht und die betreffende Handlung nicht nach gesetzlichen Vorschriften erlaubt ist, ist für die oben aufgeführten Weiterverwendungen des Materials die Einwilligung des jeweiligen Rechteinhabers einzuholen. 
Weitere Details zur Lizenz entnehmen Sie bitte der Lizenzinformation auf http://creativecommons.org/ licenses/by/4.0/deed.de.

\section{Literatur}

Abdulrahman A, Bach C (2013) How to implement marketing 2.0 successfully. Int J Bus Soc Sci 4(10):36-42

Adam T (2012) Die Bewertung von Innovationsideen - Eine empirische Analyse von Bewertungsdimensionen und sozialen Einflussfaktoren. Dissertation. Rheinisch-Westfälischen Technischen Hochschule Aachen, Aachen (http://publications.rwth-aachen.de/record/82789)

Akilan A (2015) Text mining: challenges and future directions. IEEE sponsored second International Conference of Electronics and Communication Systems (ICECS 2015). https://doi.org/10.1109/ECS. 2015.7124872

Alam I (2002) An exploratory investigation of user involvement in new service development. J of the Acad Mark Sci 30(3):250-261. https://doi.org/10.1177/0092070302303006

Arevalo M (2018) The impact of Online reviews on businesses. https://www.brightlocal.com/2017/03/15/ the-impact-of-online-reviews/. Zugegriffen: 27. Mai 2020

Aslam S, Jadoon E, Zaman K (2011) Effect of word of mouth on consumer buying behavior. MJSS 3(3):497-507. https://doi.org/10.5901/mjss.2011.v2n3p497

Bruhn M, Stauss B (2009) Kundenintegration - Forum Dienstleistungsmanagement. Gabler, Wiesbaden https://doi.org/10.1007/978-3-8349-9407-3

Charlton G (2012) E-commerce consumer reviews: why you need them and how to use them. https:// econsultancy.com/blog/9366-ecommerce-consumer-reviews-why-you-need-them-and-how-to-usethem. Zugegriffen: 27. Mai 2020

Cheung C, Sia C-L, Kuan K (2012) Is this review believable? A study of factors affecting the credibility of Online consumer reviews from an ELM perspective. J Assoc Inf Syst 13(8):618-635. https://doi. org/10.17705/1jais.00305

Data und Marketing Association (2017) Customer engagement-focus on automotive. https://dma.org.uk/ uploads/misc/5a2eb3d53bdec-customer-engagement---automotive_5a2eb3d53bd44.pdf. Zugegriffen: 27. Mai 2020

Dettweiler C (2018) Über die Nutzung von Online-Kundenrezensionen bei der Beschaffung von Business Apps. Dissertation. Universität Bayreuth, Bayreuth (https://epub.uni-bayreuth.de/4329/)

Ehrhardt A (2019) 2019 trend radar: the rating economy-results of the company survey by industry. https://www.simon-kucher.com/en/TheRatingEconomy-Overview-Companies. Zugegriffen: 27. Mai 2020 (Von Simon Kucherer und Partners: https://www.simon-kucher.com/en/TheRatingEconomyOverview-Companies. Gesehen 27. Mai 2020)

Fiedler M, Kissling M (2019) Cause and effects of fake reviews: a literature review. FDIBA Conference Proceedings 3

Haug K, Küper J (2010) Das Potenzial von Kundenbeteiligung im Web-2.0-Online-Shop: Produktbewertungen als Kernfaktor des „Consumer-Generated-Marketing“. In: Heinemann G, Haug A (Hrsg) WebExzellenz im E-Commerce: Innovation und Transformation im Handel, 1. Aufl. Gabler, Wiesbaden, S 115-134 https://doi.org/10.1007/978-3-8349-8816-4_6

Hennig-Thurau T, Gwinner KP, Walsh G, Gremler DD (2004) Electronic word-of-mouth via consumeropinion platforms: what motivates consumers to articulate themselves on the Internet? J Interact Mark 18(1):38-52. https://doi.org/10.1002/dir.10073

Heron M, Hanson VL, Ricketts I (2013) Open source and accessibility: advantages and limitations. J Interact Sci. https://doi.org/10.1186/2194-0827-1-2

$\mathrm{Hu}$ N, Bose I, Koh NS, Liu L (2012) Manipulation of online reviews: an analysis of ratings, readability, and sentiments. Decis Support Syst 52(3):674-684. https://doi.org/10.1016/j.dss.2011.11.002

Iacob C, Veerappa V, Harrison R (2013) What are you complaining about: a study of online reviews of mobile applications. Proceedings of the 27th International BCS Human Computer Interaction Conference, S 291-296 https://doi.org/10.14236/ewic/HCI2013.38

Klaas E (2019) Data Mining und Text Mining: Kleine Unterschiede, große Wirkung. Wirtschaftsinform Manag 11(4):267-269. https://doi.org/10.1365/s35764-019-00178-6

Li M, Huang L, Tan C-H, Wie K-K (2013) Helpfulness of online product reviews as seen by consumers: source and content features. Int J Electron Commer 17(4):101-136. https://doi.org/10.2753/ JEC1086-4415170404 
Lis M, Korchmar S (2013) Digitales Empfehlungsmarketing: Konzeption, Theorien und Determinanten zur Glaubwuerdigkeit des Electronic Word-of-Mouth (EWOM). Springer, Wiesbaden https://doi.org/ 10.1007/978-3-658-01008-9

Mayring P, Fenzl T (2019) Qualitative Inhaltsanalyse. In: Bauer N, Blasius J (Hrsg) Handbuch Methoden der empirischen Sozialforschung. Springer, Berlin, S 633-646 https://doi.org/10.1007/978-3-65821308-4_42

Mudambi SM, Schuff D (2010) What makes a helpful review? A study of customer reviews on amazon.com. MISQ 34(1):185-200. https://doi.org/10.2307/20721420

Neri F, Pettoni M (2008) Stalker, a multilingual text mining search engine for open source intelligence. 12th International Conference Information Visualisation. https://doi.org/10.1109/IV.2008.9

Penaflorida R (2019) The importance of automotive reviews. https://www.reviewtrackers.com/blog/ automotive-reviews/. Zugegriffen: 27. Mai 2020

Pham TD (2016) Determinants of electronic word of mouth perceived credibility: a study of cosmetic purchasing behavior. Masterarbeit, Universität Twente. https://essay.utwente.n1/70859/1/Pham_MA_ BMS.pdf. Zugegriffen: 12. September 2020

Schieber A, Kruse P (2014) Idea Mining - Wissensmanagement und Text Mining im Innovationsprozess. Dresden, S 8-35 (Arbeitspapier). Zugegriffen: 07. Oktober 2020

Schindler RM, Bickart B (2005) Published word of mouth: referable, consumer-generated information on the Internet. In: Haugtvedt CP, Machleit KA, Yalch R (Hrsg) Online consumer psychology: understanding and influencing consumer behavior in the virtual world. Routledge, Mahwah, S 35-61

Seidel LM (2013) Text Mining als Methode zur Wissensexploration: Konzepte, Vorgehensmodelle, Anwendungsmöglichkeiten. Masterarbeit, Hochschule Wismar. http://www.wi.hs-wismar.de/ cleve/ vorl/projects/da/13-Master-Seidel.pdf. Zugegriffen: 10. September 2020

Sparks BA, Browning V (2011) The impact of online reviews on hotel booking intentions and perception of trust. Tour Manag 32:1310-1323. https://doi.org/10.1016/j.tourman.2010.12.011

Usai A, Pironti M, Mital M, Mejri CA (2018) Knowledge discovery out of text data: a systematic review via text mining. J Knowl Manag 22(7):1471-1488. https://doi.org/10.1108/JKM-11-2017-0517

Wang Y, Rodgers S (2011) Electronic word-of-mouth and consumer generated content: from concept to application. In: Eastin MS, Daugherty T, Burns NM (Hrsg) Handbook of research on digital media and advertising: user generated content consumption, 1. Aufl. Information Science Reference, Hershey, S 212-231 https://doi.org/10.4018/978-1-60566-792-8.ch011

Yin RK (2009) Case study Reseach design and methods, 5. Aufl. SAGE, Thousand Oaks

Younis EMG (2015) Sentiment Analysis and Text Mining for Social Media Microblogs using Open Source Tools: An Empirical Study. IJCA 112(5):44-48 